\title{
Atypical Skin Inflammation in a 2.5-Year-Old Girl With Atopic Dermatitis
}

Krogulska A ${ }^{1}$, Sardecka I ${ }^{2}$, Skrzeczko-Kwela E ${ }^{3}$, Adamska I ${ }^{1}$ ${ }^{1}$ Department of Pediatrics, Allergology and Gastroenterology Collegium Medicum Bydgoszcz, Nicolaus Copernicus University of Torun, Poland

${ }^{2}$ Department of Pediatrics, Allergology, Gastroenterology and Nutrition, Medical University of Lodz, Poland

${ }^{3}$ Department of Dermatology, Sexually Transmitted Diseases and Immunodermatology, Collegium Medicum Bydgoszcz, Nicolaus Copernicus University of Torun, Poland

J Investig Allergol Clin Immunol 2018; Vol. 28(5): 336-338 doi: 10.18176/jiaci.0276

Key words: Atopic dermatitis. Allergic/irritant contact dermatitis. Adverse drug effects. Dithranol.

Palabras clave: Dermatitis atópica. Dermatitis de contacto alérgica/ irritativa. Efectos secundarios de los medicamentos. Ditranol.

Atopic dermatitis (AD) is a recurrent inflammatory skin disorder. Acute AD is characterized by erythematous and exudative lesions, whereas the chronic form is characterized by lichenification and crusting. According to the results of an epidemiological study conducted in Spain, AD was considered idiopathic in $58 \%$ of cases and associated with sensitization to allergens in $42 \%$ (the responsible allergens were foods in $10 \%$ and aeroallergens in $26 \%$ ) [1]. The role of the skin in the development of allergic reactions, including life-threatening ones, is increasingly significant [2].

Adverse drug reactions are a serious public health problem in that they are associated with high morbidity, socioeconomic costs, and potential fatality [3]. The gravity of this problem can be seen in the increasing incidence of accidental or intentional drug-induced toxicity in children. Drug-induced toxicity (usually through the digestive tract, but also through the skin) is a common problem and one requiring continuing education.

A 2.5-year-old girl with $\mathrm{AD}$ was referred to the outpatient allergology clinic with intensified, atypical inflammatory skin lesions and accompanying pruritus.

The history showed that the girl had been diagnosed with $\mathrm{AD}$ at the age of 2 months. She was therefore given standard treatment for moderate $\mathrm{AD}$ and recommended a dairy-free diet, which led to partial improvement. At 14 months of age, she underwent allergy testing (sIgE with food and inhalant allergens was negative; an atopy patch test with milk was positive) and an open food challenge with cow's milk. Given the observed exacerbations of $\mathrm{AD}$, she returned to a dairy-free diet. She had typical symptoms of AD on her wrists, popliteal areas, and neck, although during the 4 months before her most recent consultation, she did not experience flare-ups. In the opinion of the parents, the recent exacerbations had no connection with the consumption of food or the action of other external factors. Despite the presence of skin lesions, no other symptoms were observed at admission. 

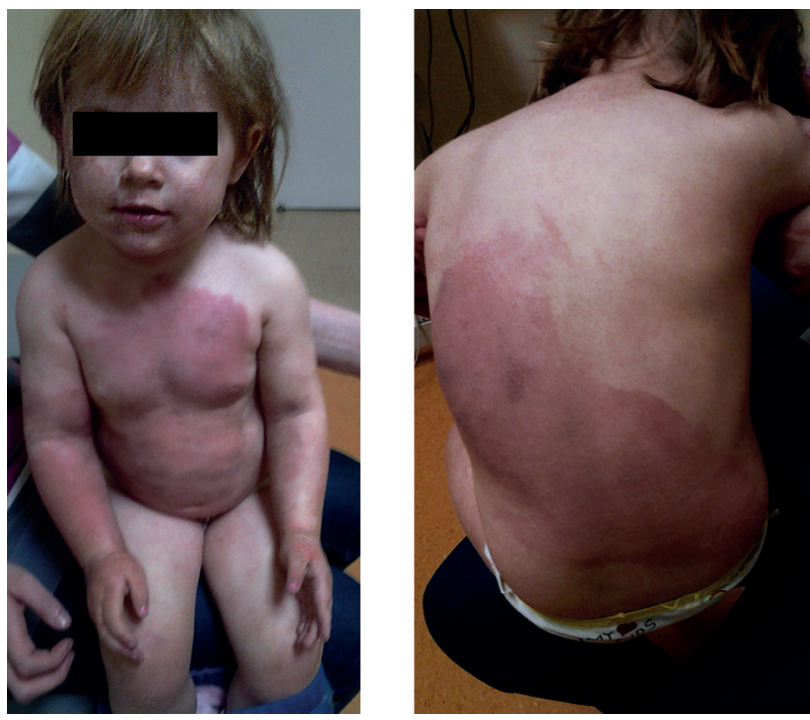

Figure. Figure, A and B. Intensely red inflamed areas.

The patient was in good health, the physical examination revealed the presence of confluent inflammatory patches with a well-defined edge accompanied by severe itching on the cheeks, chest, stomach, back, groins, and upper limbs (Figure, A and B). Our initial suspicion was exacerbation of $\mathrm{AD}$; however, the skin erythema was too intense, with an atypical shape and clear margins. In urticaria, skin changes are accompanied by characteristic itchy wheals. Erysipelas was ruled out, since the skin changes were too extensive, multifocal, and with no general symptoms or inflammatory markers. No data in the history indicated exposure to cosmetics, chemical substances, plants, or sunlight.

Further information from the child's parents helped us to establish the diagnosis. On the third day of hospitalization, the parents revealed that the child's grandmother, who was under treatment for psoriasis, mistakenly applied her dithranol 2\% ointment to the child's skin and did not inform anyone about this.

The clinical data suggest irritant contact dermatitis caused by dithranol in a child with AD. With appropriate treatment and monitoring of the atopic changes, the patient's skin returned to normal 4 weeks after the accidental exposure to dithranol.

Medical errors, including incorrectly administered drugs, are the third leading cause of death in the USA [4]. As many as $44 \%$ of all cases of toxicity in children below 5 years of age are caused by drugs used in medical care, especially those prescribed to their caretakers [5]. The most common route of drug toxicity is through the gastrointestinal tract; transdermal drug toxicity is uncommon. The predisposing factors for toxicity in children, even if induced via skin contact, are differences in metabolism and immaturity of anatomical barriers in the developing child. Chemical substances applied to a child's skin can induce both topical and systemic effects.

Dithranol is an organic chemical compound from the polyphenol group. It was originally extracted from the araroba tree and remains one of the most widely used and effective treatments for psoriasis. According to De Jager et al [6], dithranol is used in children at low concentrations $(0.016 \%$ $0.625 \%$ ). In adults, it is usually applied at concentrations ranging from $0.1 \%$ to $5 \%$. The present case involved $2 \%$ dithranol ointment. Around 4\% of patients stop using the drug owing to adverse effects. Reported adverse effects after topical administration of dithranol include burning sensation, irritation, and redness of the skin [7]. The main difficulties with its use are irritation, staining of the skin, and allergic contact dermatitis. Patch testing is important in patients previously described as dithranol-intolerant [8-10]. However, the irritant potential of dithranol hampers patch testing performed to differentiate between allergy and irritation. Moreover, the threshold for detection of contact hypersensitivity may theoretically be above the irritant threshold. It has been suggested that dithranol allergy is more common than generally suspected and that patients who do not tolerate dithranol should undergo patch testing with the allergen [8]. While a positive reaction may only be obtained in a minority of patients, those with a negative result, and therefore a presumably irritant response, could be treated more confidently with lower concentrations rather than having to discontinue a potentially useful treatment. In contrast, the minority who are truly allergic to dithranol should avoid this compound in all its formulations. Loffler et al [9] showed that a pronounced skin reaction to rather low concentrations of dithranol is common, although it is not clear whether this represents an allergic or irritant reaction. The authors concluded that increased reactivity to dithranol most likely reflects genuine increased skin susceptibility, rather than an allergic response.

When applied to a large skin area, dithranol might induce toxicity and lead to symptoms such as those of central nervous system disturbances, kidney damage, and methemoglobinemia. The drug should be used with caution in children with psoriasis. Physicians should make every effort to inform patients about the prevention of drug-induced toxicity.

\section{Funding}

The authors declare that no funding was received for the present study.

\section{Conflicts of Interest}

The authors declare that they have no conflicts of interest.

\section{References}

1. Martorell Aragonés A, Félix Toledo R, Martorell Calatayud A, Cerdá Mir JC. Epidemiologic, clinical and socioeconomic factors of atopic dermatitis in Spain: Alergológica-2005. J Investig Allergol Clin Immunol. 2009;19 Suppl 2:27-33.

2. Liccardi G, De Falco F, Gilder JA, D'Amato M, D'Amato G. Severe systemic allergic reaction induced by accidental skin contact with cow milk in a 16-year-old boy. A case report. J Investig Allergol Clin Immunol. 2004;14:168-71.

3. Faria E, Rodrigues-Cernadas J, Gaspar A, Botelho C, Castro E, Lopes A, et al. Drug-Induced Anaphylaxis Survey in Portuguese Allergy Departments. J Investig Allergol Clin Immunol. $2014 ; 24: 40-8$ 
4. Makary M, Daniel M. Medical error - the third leading cause of death in the US. BMJ. 2016;353:i2139.

5. Burghardt LC, Ayers JW, Brownstein JS, Bronstein AC, Ewald MB, Bourgeois FT. Adult Prescription Drug Use and Pediatric Medication Exposures and Poisonings. Pediatrics. 2013;132:112.

6. De Jager ME, de Jong EM, van de Kerkhof PC, Seyger MM. Efficacy and safety of treatments for childhood psoriasis a systematic literature review. I Am Acad Dermatol. 2010;62:1013-30.

7. Bruner $C R$, Feldman $S R$, Ventrapragada $M$, Fleischer $A B$ Jr. $A$ systematic review of adverse effects associated with topical treatments for psoriasis. Dermatol Online J. 2003:9:2.

8. Burden $A D$, Stapleton M, Beck MH. Dithranol allergy: fact or fiction? Contact Dermatitis. 1992;27:291-3.

9. Loffler H, Effendy I, Happle R. Skin susceptibility to dithranol: contact allergy or irritation? Eur J Dermatol. 1999;9:32-4.

10. Holguín-Gómez L, Sastre Domínguez J. Occupational Contact Dermatitis in Spain. J Investig Allergol Clin Immunol. 2017;27:134-6.

1 Manuscript received December 11, 2017; accepted for publication May 22, 2018.

Aneta Krogulska

Department of Pediatrics, Allergology and Gastroenterology

CM Bydgoszcz, NCU Torun

ul. M. Curie Skłodowskiej 9, 85-094 Bydgoszcz, Poland

E-mail: anetkrog@poczta.onet.pl 